\section{Superconducting regions in lysozyme}

from a Correspondent

Great interest was aroused at the Dielectrics Society annual meeting by Herbert Fröhlich's report of some experiments which show that an aqueous solution of the enzyme lysozyme contains regions which are superconducting. The report (now published: Ahmed, Calderwood, Fröhlich and Smith, Phys. Lett., 53A, 129; 1975) can be summarised thus.

Magnetic fields of order 600 Gauss were found to bring about very large changes in the dielectric constant of aqueous lysozyme solutions. Since for this to happen the magnetic field must be able to overcome ionic thermal fluctuations, the magnetic energy must act over a volume of the order of a million lysozyme molecules, which indicates the existence of a cooperative phenomenon capable of increasing the magnetic susceptibility in a

of chemically distinct lipids present in that membrane. That goes against the considerable weight of experimental evidence suggesting specific interactions between particular proteins and lipid classes, with at least a ring of lipid bound relatively tightly to the protein in a long-lived interaction. So it seems that whereas the bulk of the lipid could be diffusing as fast as lipid in simple lipid bilayers, there might also be some lipid in contact with protein and exchanging only slowly with the bulk lipid.

In a recent paper, Warren et al. (Nature, 255, 684; 1975) explore this possibility of relatively long-lived lipidprotein interactions with their simplified lipid-protein system, containing the $\left(\mathrm{Ca}^{2+}-\mathrm{Mg}^{2+}\right)$ ATPase from sarcoplasmic reticulum. They have found that the activity of the protein is maintained as long as there are 30 or more phospholipid molecules for each protein molecule: with a smaller proportion of phospholipid there is an irreversible loss of activity. It has been suggested therefore that these 30 lipid molecules form a single shell of phospholipid bilayer around the protein, protecting it from denaturation. Further experiments have then shown that cholesterol, which on its own leads to a reversible loss of activity, cannot normally penetrate this lipid annulus. If, as seems likely, this proves to be a general property of membrane proteins, then the organisation of lipids within the membrane will be by no mean homogeneous. Each protein will be surounded by a lipid microenvironment, the structure of the magnetic field.

This measurement was therefore made, and a $1 \%$ increment in bulk susceptibility was found on application of the 600 Gauss field. This is ten thousand times larger than the effect for an ordinary diamagnetic material and its disappearance about 800 Gauss indicates that it represents a Meissner effect in superconduction. Complicated temperature and concentration effects were found, including temperature hysteresis. It was postulated that in solution the ions form a layer structure, giving rise to an a.c. Josephson effect, that is, to the electric vibrations in biomolecules recently proposed by Fröhlich and reported in the Soviet literature. Physiological effects of magnetic fields would seem to be given physical respectability if a Meissner effect exists in a biomolecular solution.

protein and the nature of the microenvironment together determining the functioning of the protein. Only the bulk lipid will be free to diffuse within the membrane, and around each protein there will be an immobilised lipid annulus, whose composition will be determined by the structure of the protein. Even in the transitory world of membranology, not everything is in a state of flux.

\section{Mammalian development, with chicks and newts}

\section{from Gillian M. Morriss}

The Ciba Foundation held a symposium on "Embryogenesis in Mammals" on June 16-19. The proceedings will be published by Associated Scientific Publishers.

THE symposium was held just ten years after the symposium on preimplantation states of pregnancy-ten years which have seen a tremendous creative surge in the field of vertebrate experimental embryology. With congenital anomalies an increasing medical problem, much of this creative energy has been applied to studying normal and abnormal postimplantation mammalian development. Whole embryo and organ culture techniques have contributed atly to the accessibility and ma dity of the systems under investigation; however, in comparison with the work on other vertebrates, mammalian embryology appears to have embarked on an obstacle course beset with many frustrations. Thus it was fitting that two presentations on recent advances in avian and amphibian development were included in the symposium, and that four additional non-mammalian embryologists were present as discussants.

\section{Death of a germ layer}

More than one established dogma was challenged by the participants. The first was the demonstration by $\mathbf{R}$. L . Gardner (University of Oxford) that the early differentiation of inner cell mass into 'endoderm' (hypoblast) and 'ectoderm' (epiblast) does not signify the establishment of cell lineages loyal to the historical concept of germ layers. The most important determinative factor in the differentiation of preimplantation and implanting embryos is cell position; thus chimaeras can be constructed whose primary endoderm-derived cells are detectably different from the rest of the embryo. Examination of such embryos at $15 \frac{1}{2}$ days showed that the whole embryo is derived from the so-called ectoderm. Other experiments demonstrated that even the germ cells are ectodermal in origin.

N. Skreb (Faculty of Medicine, Zagreb), describing a study carried out in collaboration with B. Levak-Svajger and A. Svajger (see J. Embryol. exp. Morph., 32, 445-467; 1975), showed that, when transplanted to a site under the adult kidney capsule, embryonic ectoderm retains the capacity to differentiate into all embryonic structures (including gut) up to the primitive streak stage, though not later. Endoderm alone was always resorbed, but this may simply be a further demonstration of the essential interactive role of adjacent mesenchyme and intercellular matrix in epithelial differentiation. Nicole Le Douarin (Université de Nantes), for instance, showed that grafts of portions of avian pharyngeal endoderm will not differentiate in the absence of mesoderm. The major part of her paper concerned that unique piece of ectoderm, the neural crest. Her elegant studies on quail-chick transfers are too well known to need description here (see, for instance, Devl Biol., 41, 162-184; 1975); as the chairman, Anne McLaren (University College, London) remarked, they are a source of envy to mammalian embryologists. A further characteristic of the primitive endoderm is its slow proliferation: M. Snow (University College, London) calculated that in the primitive streak stage mouse embryo, the cell cycle time in the endoderm is more than $15 \mathrm{~h}$, compared to $6 \frac{1}{2}$ and 